\title{
A Feathering Effect Detector based on Local Average and Effective Values
}

\author{
André L. Martins \\ University of São Paulo \\ 400, Trabalhador São-carlense \\ São Carlos - SP - Brazil
}

\author{
Evandro L. L. Rodrigues \\ University of São Paulo \\ 400, Trabalhador São-carlense \\ São Carlos - SP - Brazil
}

\author{
Maria Stela V. Paiva \\ University of São Paulo \\ 400, Trabalhador São-carlense \\ São Carlos - SP - Brazil
}

\begin{abstract}
Feathering effect is a typical phenomenon present on video frames generated by interlacing process. This effect is easily identified by the human eye when objects in motion are present in the scene, since it causes visual discomfort. This paper presents an algorithm to detect feathering effect on interlaced video frames, identifying areas on the image to be corrected by another algorithm at a later stage. Before performing de-interlacing processes itself, like motion compensation or ELA, to identify the defected regions can improve the general performance. The proposed algorithm is a preliminary stage on whole de-interlacing process; through the precise indication of the defective regions, the deinterlacing algorithm is able to work more efficiently, eliminating the scanning of whole image pixels. Test results confirmed that the basic principle of the algorithm is correct, reaching almost $100 \%$ of detecttion accuracy for the analysed images domain, and is invariant to image resolution, object size, motion direction and contrast to background.
\end{abstract}

\section{General Terms}

Video interlacing, motion adaptive, motion compensation, motion estimation, ELA.

\section{Keywords}

Feathering effect, de-interlacing.

\section{INTRODUCTION}

As described by Martins [1], digital TV stations must generate video transmission for interlaced field scan. On interlaced pattern, each video frame is split into two sets of lines, named the "fields." For each frame, odd-numbered lines are arranged in a field called the "odd field," and the same for even lines. Thus, it is necessary that the TV sets perform the conversion process from interlaced to progressive; this process is called "de-interlacing."

De-interlacing generates distortions in the image, known as "video artifacts." Many distortions generated by the process of de-interlacing are caused by moving objects in the image.

Advanced de-interlacing algorithms are able to identify regions of the image where there is movement, and apply the so-called "motion compensation"; such algorithms require the determination of true-motion vectors, like described by Bellers [2], or other advanced de-interlacing processes, like ELA, described originally by Doyle [3] and deeply explored by Jeong [4].

In this paper, the development of a feathering effect detection algorithm is presented by the strategy that identifies "featherlike" structures (or "comb-like" structures) by evaluating the periodical wave behavior of the defects. The proposed method contributes to increase the performance of de-interlacing methods executed a posteriori by delivering them the coordinates of areas with defects. De-interlacing methods could take advantage of the proposed algorithm and improve their performance

In the next section, a more detailed description of what typically causes feathering effect and its characterization are presented.

\section{FRAME INTERLACING AND FEATHERING EFFECT}

In simple de-interlacing method, basically ordering of lines occurs; odd field lines are interspersed with the even field lines, forming a complete progressive frame (with the lines in numerical sequence. This de-interlaced frame presents video artifacts in regions where there is motion from one field to another. Because the temporal mismatch between the fields, and if there is motion of objects between the moments of acquisition of two fields, the reorganization of lines generates a double image effect, or "feathering effect" on parts of objects or people who move from one field to another. The term "feathering effect" has been already used, described and treated by some authors, like Chang [5], Jeon [6], Fan [7] and Sreekanth [8].

For the human eye, feathering effect looks like two images in one, and the edges of objects appear like a bird feather, or even like a comb. A representative of the effect can be seen on Figure 1, extracted from PC Magazine [9].

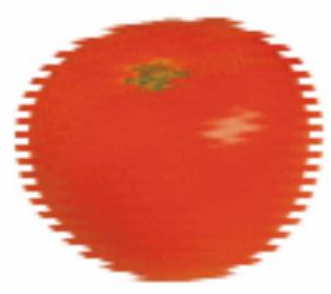

Figure 1: The feathering effect gives a feather like or comb like appearance to objects in motion (source: PC Magazine OnLine)

\subsection{Feathering effect characterization}

This effect worsens the visual perception as the speed of the objects increases. Beyond that, based only on subject feeling, it is expected that the effect and its discomfort for the human eye be higher for lower resolutions, object size and intensity level of pixels to the image background. It is easy to observe the defects on light reflections and details on internal edges in the objects.

Some authors proposed feathering identification embedded on de-interlacing methods; a method to identify feathering effect 
was proposed by Chen [10], based on difference of pixel level between original pixels and estimated pixel lines through interpolation.

In the next section, the details of the proposed algorithm and the strategy used for performance verification are presented.

\section{FEATHERING EFFECT IDENTIFICATION}

One of the main motivations during the development of the proposed method was to identify the neighboring conditions where the human eye feels discomfort, considering some characteristics and parameters as: object speed from one frame to another, size of the moving objects, object contrast to image background, motion direction and object shape.

The visual repetitive nature of the phenomenon takes us to think on the similar behavior of a periodic waveform; is well known that a periodic waveform can be characterized by representative calculations like average value and effective value. Periodic waveforms oscillating over zero presents an average value of zero. From electrical engineering point of view, this waveform profile presents "zero DC value".

\subsection{Base principles}

Considering an interlaced frame with a single object in motion, that object was isolated followed by a simple analysis. A hypothetical region of an interlaced frame is presented on Figure 2, which was divided into pixels. The moving object in the left side of figure has pixels market with value " 1 ". The board on the right side indicates the difference between lines, pixel by pixel, line by line.

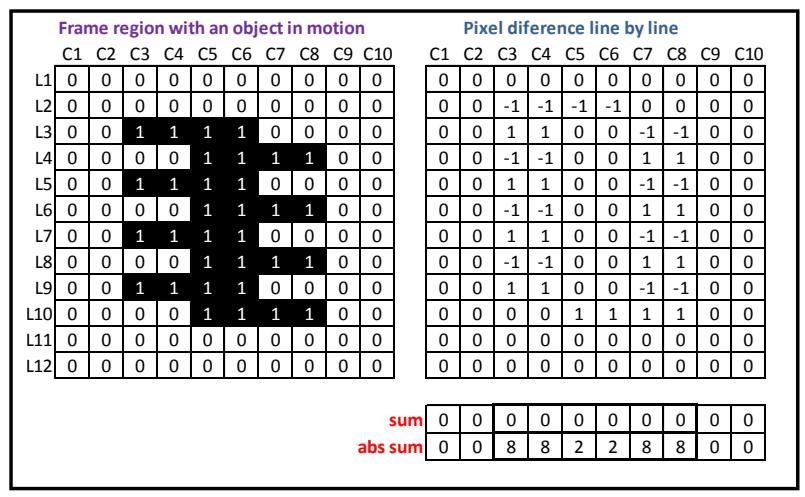

Figure 2: An interlaced frame region with an object in motion, the difference between lines, the sum and absolute sum.

Considering the board of pixel differences, a simple summation of all lines is performed, and also the summation of absolute values; interesting behavior can be observed on the regions where the feathering effect is present: higher values for absolute summation, combined to lower values for simple summation. These numbers bring us the idea of: when the feathering is present, there is a periodic wave present on the difference board. More than that: for certain frequencies, the wave has low average value and high effective value, like a periodic wave oscillating over zero reference.

\subsection{The proposed algorithm}

In the proposed algorithm, the average and effective values are calculated considering the spatial period, in terms of pixels.

Three fields are needed to run the detector algorithm, and the value of the pixels in the same coordinates are taken from field $n, n+1$ and $n+2$. Fields $n$ and $n+2$ are the same parity, and field $n+1$ is opposite parity. Frame $n+2$ is necessary only to confirm differences caused by object motion, and does not participate on other calculations.

The evaluation process evolves first the definition of a delimited area for local evaluation. The dimension (in terms of pixels) of this area deeply depends on which spatial period the defects present.

Once the spatial period is defined, the calculation of local average value (AVG) was used as a first quantification for comparing. The calculation of the local AVG value is presented in (1).

$$
A V G=\frac{\sum_{\vec{x} \in B(\vec{X})}(F(\vec{x}, n)-F(\vec{x}, n+1))}{T}
$$

where:

- $\quad A V G=$ local average value;

- $\vec{x}=$ pixel coordinates in the field $n$;

- $\quad B=$ selected block in the field $n$;

- $\vec{X}=$ block coordinates in the field $n$;

- $\quad F=$ pixel level;

- $\quad n=$ current field number;

- $T=$ spatial period in pixels;

It is also necessary to calculate the local effective value (EFT) for the region. The calculation of the local EFT value is presented in (2).

$$
E F T=\frac{\sum_{\vec{x} \in B(\vec{x})}|F(\vec{x}, n)-F(\vec{x}, n+1)|}{T}
$$

where:

- $\quad E F T=$ Effective value;

- $\vec{x}=$ pixel coordinates

- $B=$ selected block in the field $n$;

- $\quad \vec{X}=$ block coordinates in the field $n$;

- $\quad F=$ pixel level on original frame;

- $n=$ current frame number;

- $T=$ spatial period in pixels;

When the value of EFT is high and the value of AVG is low, it is considered that the periodic behavior of a wave is present, and the region is marked as positive, or, in other words, the feathering effect is identified. How to consider that EFT is high and AVG is low? These values were found empirically after running tests with the proposed algorithm, considering some parameters that could impact the visual perception in different levels.

Besides this basic evaluation, another phenomenon needs to be analyzed: when the wave behavior is not caused by feathering, but the image really presents regions like textures, the detector could mark wrongly these regions. To avoid this inconstancy, the $3^{\text {rd }}$ frame is necessary $($ frame $n+2)$. Here a procedure to detect non motion regions and correct wrong identifications is described.

The $3^{\text {rd }}$ frame, or " $n+2$ " frame, is used to avoid wrong identification caused by specific textures on image that fits the $\mathrm{AVG}$ and EFT requirements. The field $\mathrm{n}$ is compared to next field with same parity $(n+2)$ to confirm motion between 
frames. If no differences are detected, no motion is present, so the region is unmarked and ignored.

This process occurs block by block, until the entire video field is swept and all the missing lines are estimated.

\subsection{Performance evaluation of the proposed algorithm}

To estimate correctness, false positive and false negative rates detected by the proposed algorithm, artificial interlaced fields were created with controlled defects inserted on them. The procedure adopted was as follows:

a. Create a frame $\mathrm{n}$ with different objects on it;

b. Make a copy of this frame, shifting some selected objects; this frame is $\mathrm{n}+1$;

c. Remove the odd-numbered lines from frame $n$ and even-numbered lines of from frame $\mathrm{n}+1$, producing the artificial odd and even fields;

d. The two fields (odd and even) are combined, generating an artificial interlaced frame

e. With the interlaced frame in hands, and knowing in advance where the defects are, the detection algorithm is put into action.

The produced interlaced frame is subjected to the proposed feathering effect detection algorithm. It dismounts the frame into even and odd frames, as done with real video frames.

As illustration, two artificial frames created using this process steps are presented Figure 3 .

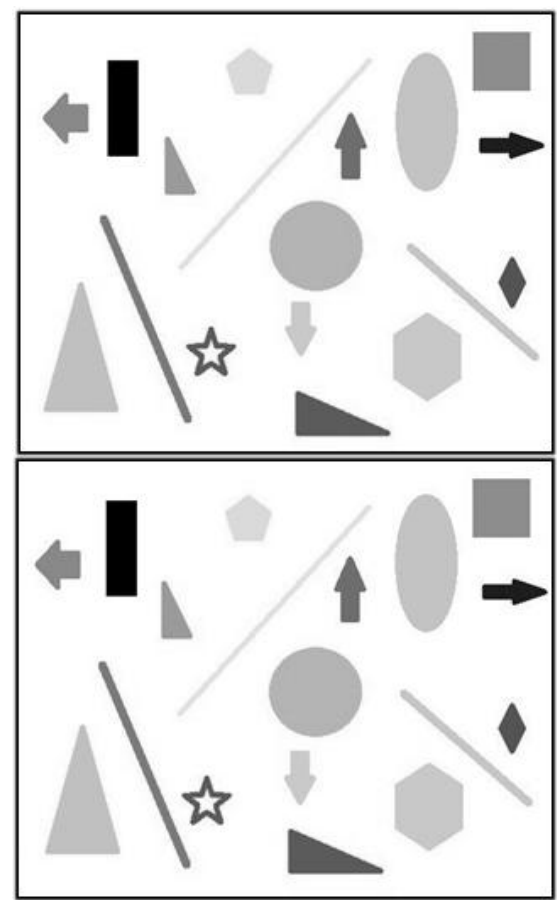

Figure 3: Artificially generated frames

The two generated frames have slight differences to simulate motion on some objects, besides other stationary objects; the differences can be easier observed by calculating the difference of the frames, as can be shown on Figure 4.

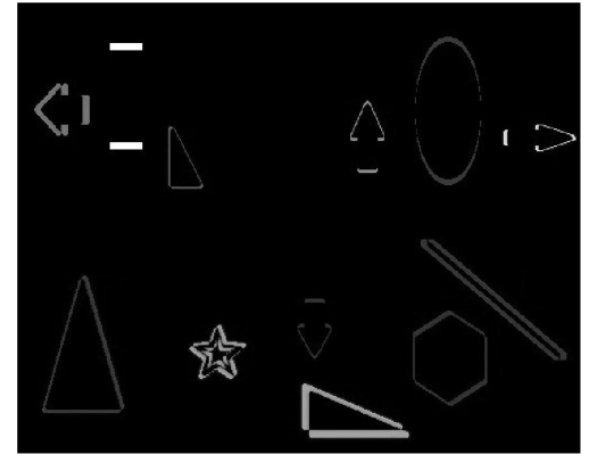

Figure 4: Frame difference id calculated to verify objects motion.

It is natural that stationary objects do not appear on frame difference.

Continuing with the process illustration, the next steps are presented in the following figures.

Odd and even fields generated by using these process steps are presented Figure 5.
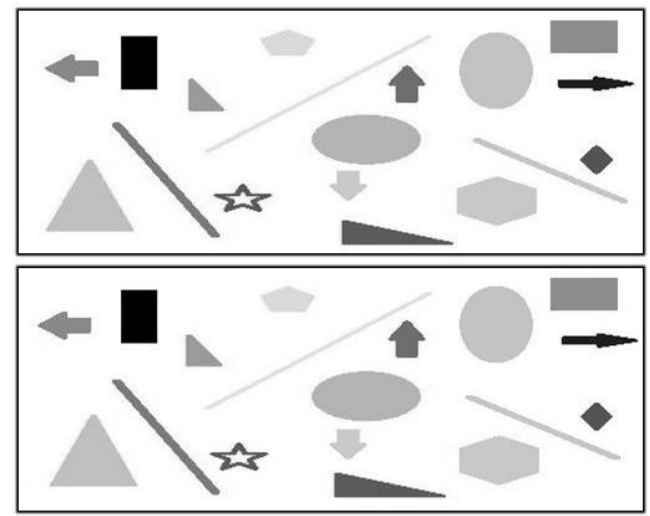

Figure 5: Artificial odd (n) and even $(n+1)$ fields.

The two fields (odd and even) are combined, generating an artificial interlaced frame, as can be shown on Figure 6.

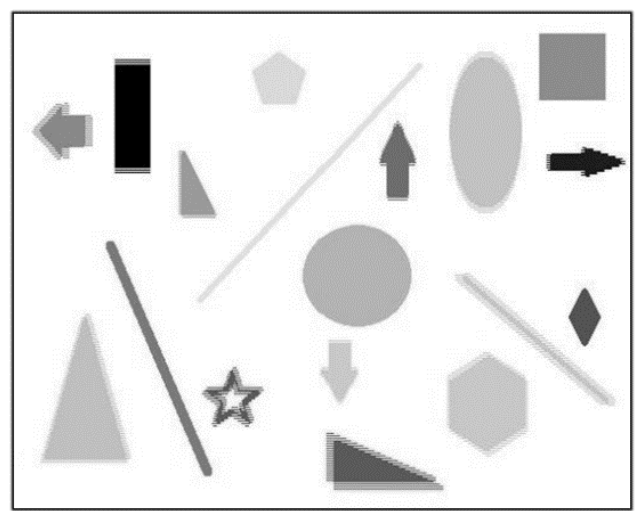

Figure 6: Artificial interlaced frame

It is clear that some objects present feathering effect, as they are in motion from one field to another. In the same way, some objects do not present defects, as they are not in motion.

As the defects are inserted purposely, they can be previously identified by visual inspection. 
Other artificial frames were created reinforcing specific characteristics that could change level of defects and the perception by human eye.

The artificial frame presented on Figure 7 was used to evaluate the algorithm performance with different object sizes combined to different contrast to image background. This image was shifted by 1 up to 4 pixels, and the same process to create artificial interlaced frames was done; each pair of frames was submitted to the proposed algorithm.

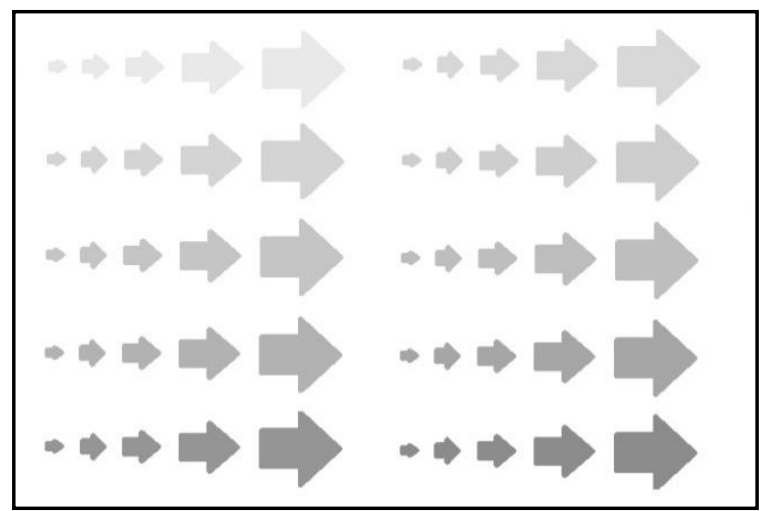

Figure 7: Artificial 720x480 pixels frame for testing; the same object in different sizes and different contrast to back.

The detection threshold level for effective and for average value were adjusted looking for the best results possible, and always considering the threshold level for average value as a fraction of effective value.

The algorithm was set to run varying the spatial period from 2 up to 14 pixels and the performance was evaluated for each situation.

The proposed algorithm has been implemented in the MatLab platform [11].

In the next section, the results of the performed tests are presented.

\section{TEST RESULTS}

Among multiple tests conducted, part of the results in applying the proposed algorithm on artificial frames and real video files are presented here.

After applying the algorithm on different images, with different resolutions, the best results were obtained with a spatial period of 6 pixels.

Regarding detection threshold level for effective value, the best results were obtained when adjusted for 0.3 (considering pixel intensity from 0 to 1 ), and the threshold for average value adjusted for values lower than $60 \%$ of effective value.

The effective value threshold is considered as the sensitivity level for detection, since the overall detection rate can be controlled by changing only this single parameter.

The proposed detector generates a frame indicating the regions where the defects were identified. To illustrate the output generated by the proposed detector, the frame resulting from feathering detection algorithm applied to frame on Figure 6 is presented on Figure 8.

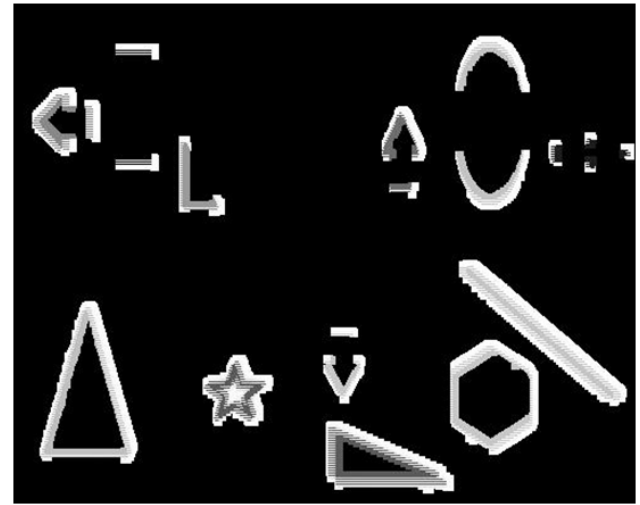

Figure 8: Frame result from proposed feathering effect detection algorithm.

In this last example, it is clear on Figure 8 that the algorithm was able to identify all feathering that is present in the image.

Real video files were also submitted to the proposed algorithm, as can be verified on Figure 9. In this frame, there are several areas where feathering is present. The algorithm detected basically most of the defects.

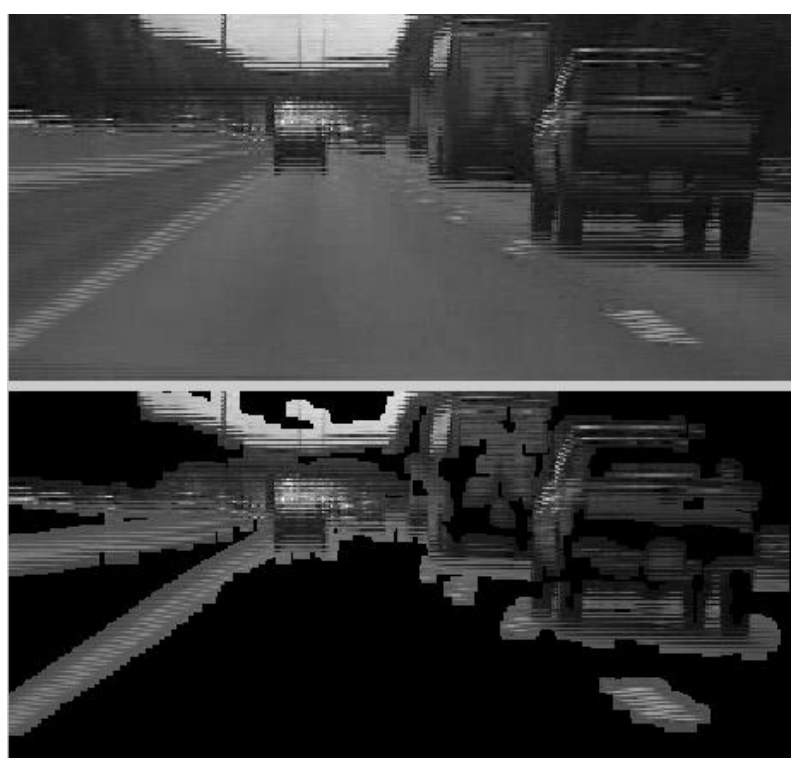

Figure 9: Real video frames but artificially interlaced and the defects detected by the proposed algorithm.

A frame with a group of a simple object moving to the right side can be verified on Figure 10, with different motion shifts, and another group moving to the up side, varying the shift from 1 to 8 pixels. The feathering effect increases as shift is bigger. For shift of 1 pixel to the right, the algorithm detected feathering on the sharp corners. 


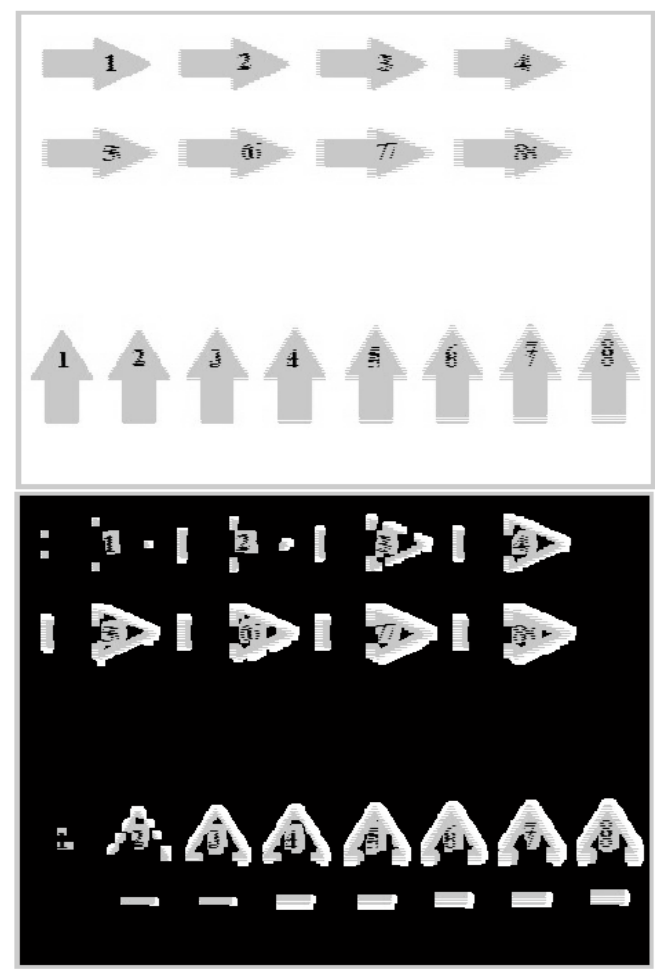

It could be verified on Figure 10 a frame with a group of a simple object moving to the right side, with different motion shifts, and another group moving to the up side, varying from 1 to 8 pixels. The feathering effect increases as shift is bigger. For right shift of 1 pixel, the algorithm detected feathering on the $90^{\circ}$ corners.

An interesting visual phenomenon can be verified on behavior of accumulated effective value over the entire image, as shown on Figure 11. The amplitude profile of accumulated value look like walls higher and higher as the motion shift becomes bigger.

Figure 10: An artificial interlaced frame with a simple moving object, varying shift from 1 to 8 pixels to the right side and to up side (left image) and a frame produced by

the proposed method, indicating the regions where feathering is present

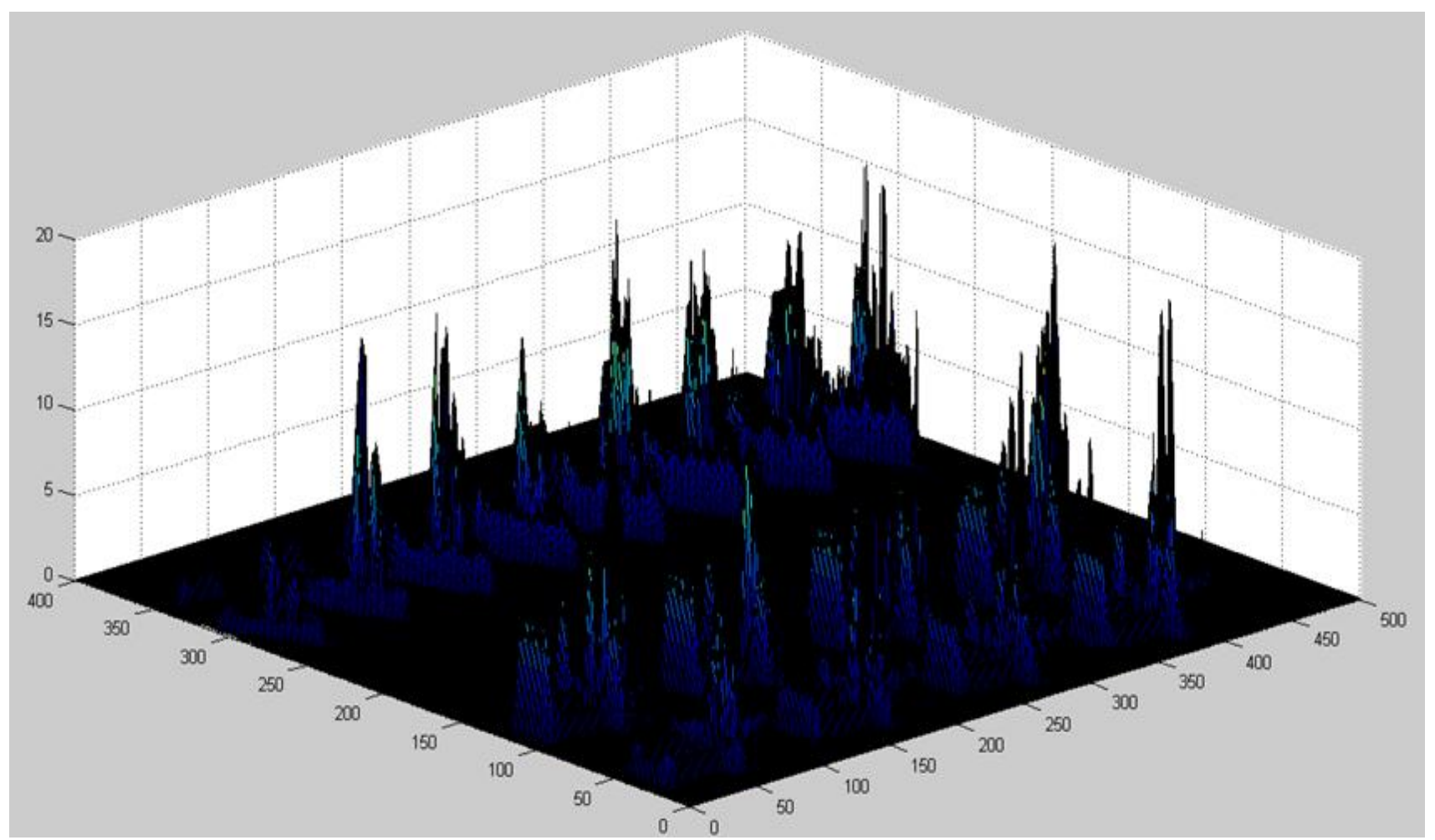

Figure 11: 3D graph showing effective accumulated value.

In the same way, the visual behavior of average accumulated value from frame on Figure 10 can be observed on Figure 12, where the periodic waveform can be observed on the areas with feathering. 


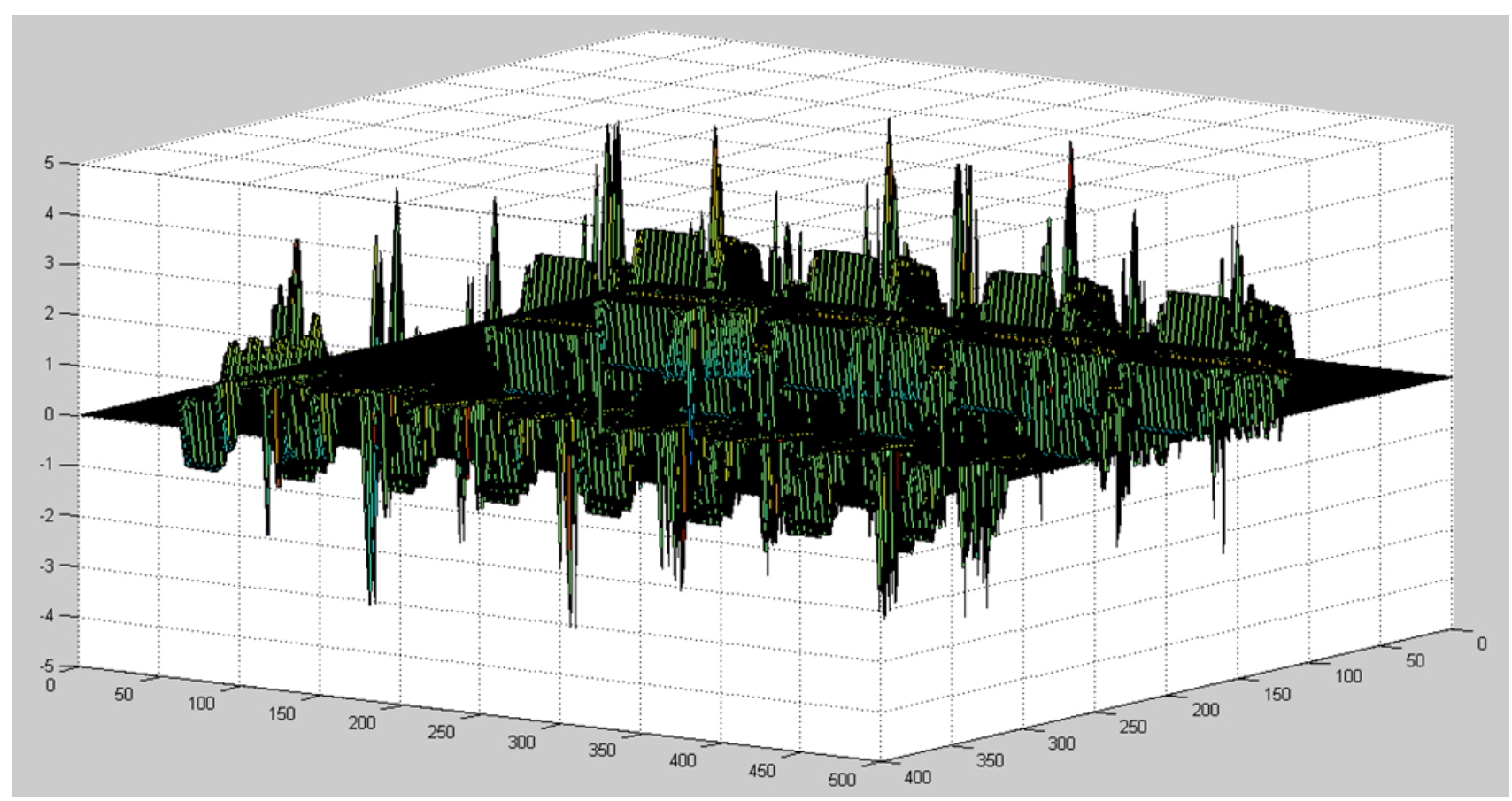

Figure 12: 3D graph showing average accumulated value, and its periodic oscillating nature.

Results obtained by shifting frame presented on 7 are presented on Figure 13, Figure 14, Figure 15 and Figure 16. The same frame was shifted from 1 to 4 pixels to create frame " $n+2$ ", and both submitted to the proposed algorithm. In this test, contrast level to background and object size did not have great influence on detection; however, motion shift parameter had much higher influence.

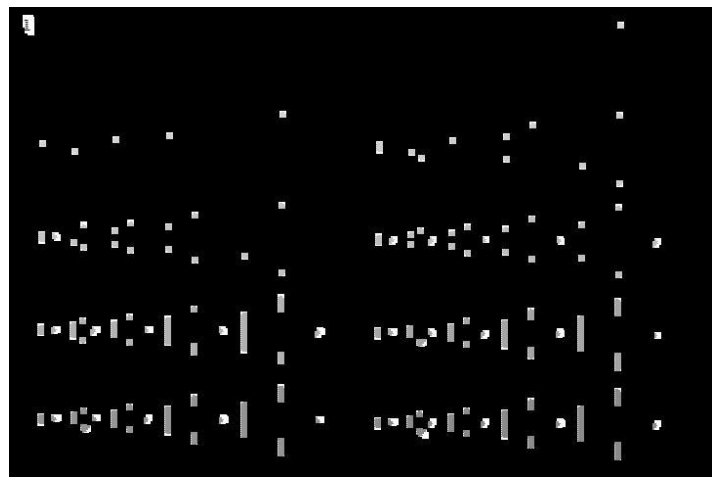

Figure 13: Motion of 1 bit to right, different size, different contrast objects.

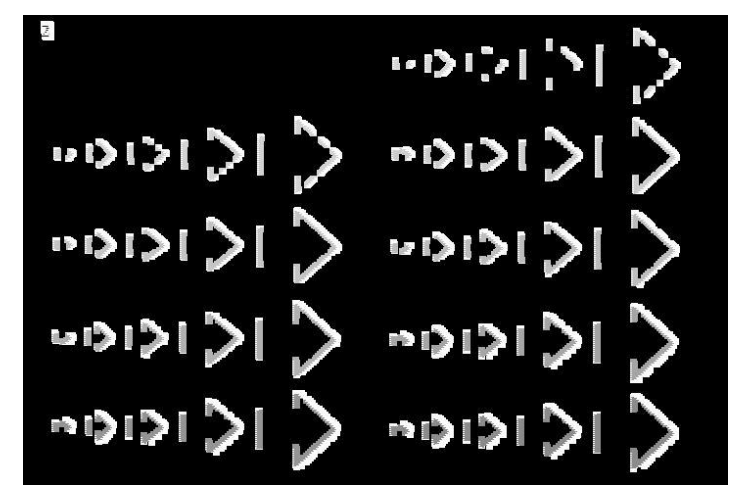

Figure 14: Motion of 2 bits to right, different size, different contrast objects.

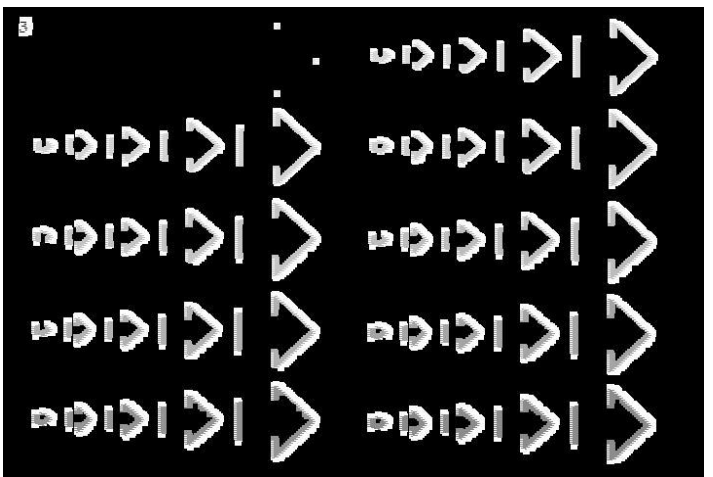

Figure 15: Motion of 3 bits to right, different size, different contrast objects

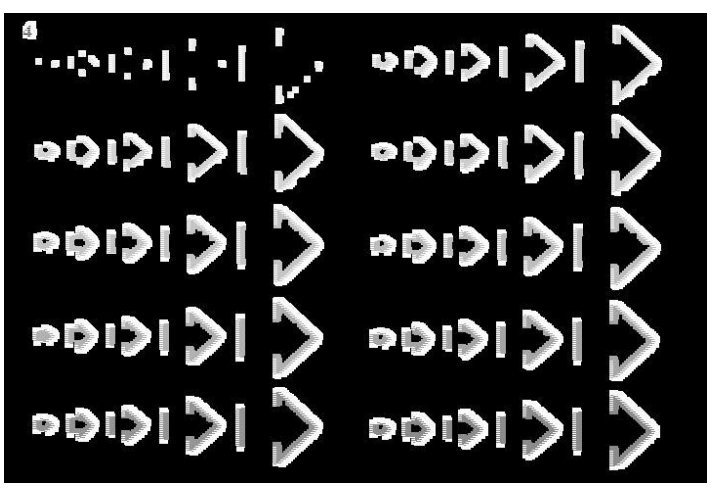

Figure 16: Motion of 4 bits to right, different size, different contrast objects.

To evaluate the performance regarding image resolution, some HD frame (1280x720 pixels) were created in the same way as described before, and submitted to the proposed algorithm. One of these tests can be seen on Figure 17. Very good results were obtained; it must be considered that spatial period of 6 pixels was fixed for all tests. 


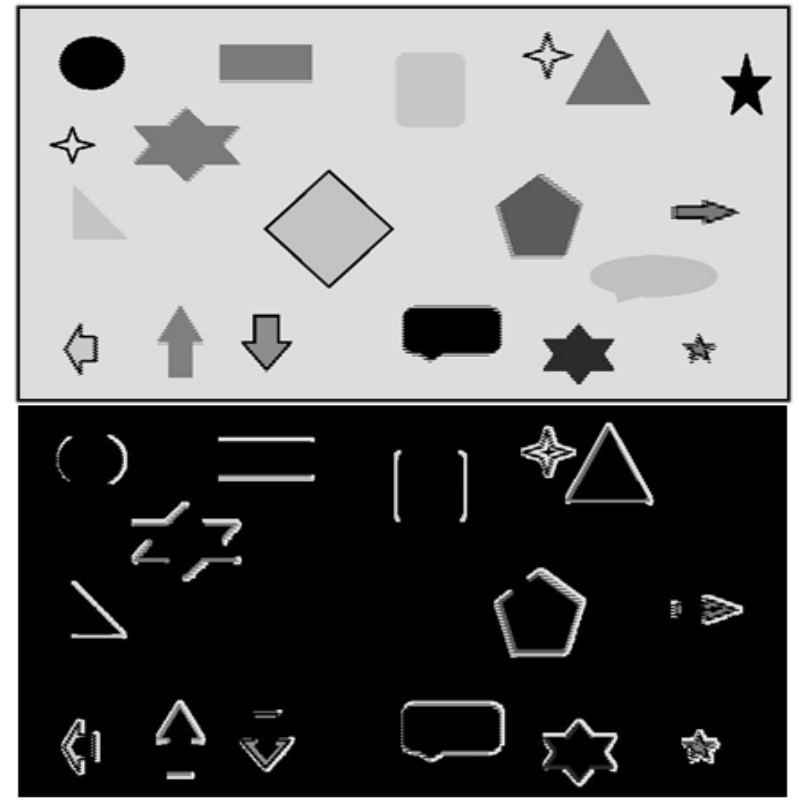

Figure 17: Artificial HD frame (1280x720 pixels)

Continuing with the evaluation of performance regarding image resolution, some full HD frames (1920x1080 pixels) were created in same way as described before, and submitted to the proposed algorithm. One of these tests can be seen on Figure 18.

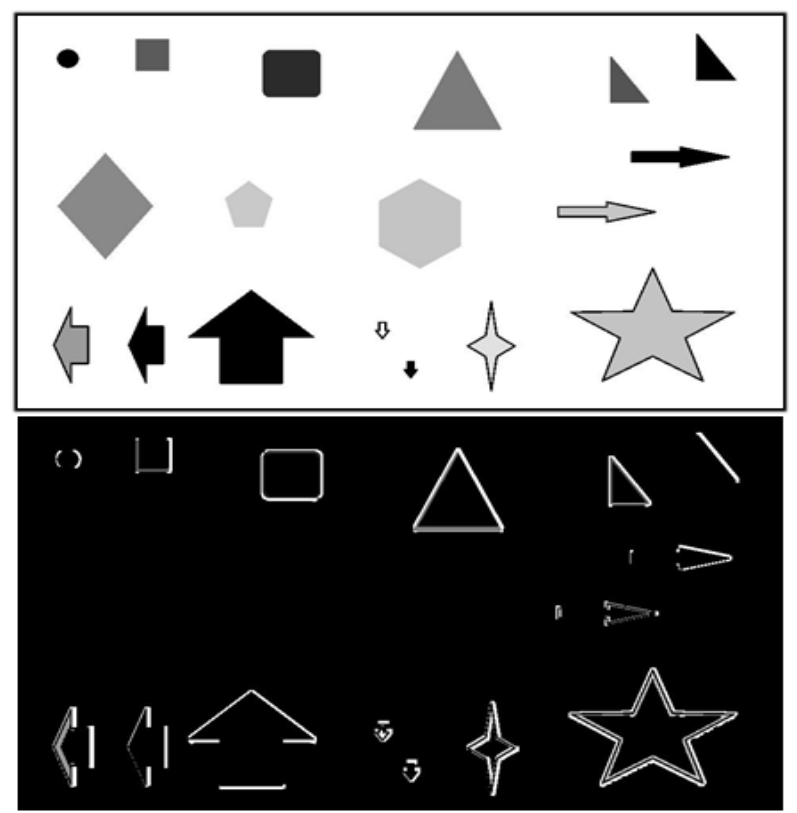

Figure 18: Artificial full HD frame (1920x1080 pixels)

Even for slightly defects on full HD frame, the proposed algorithm was able to detect all of them.

On Figure 19 it is possible to observe a frame with the same object, same size, but with different contrast to back and different motion shift in pixels to down side.

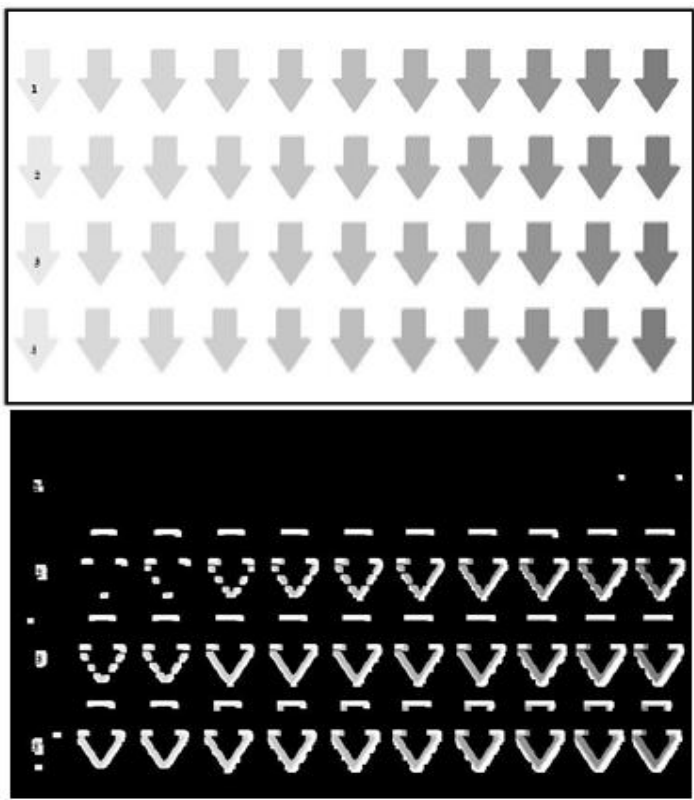

Figure 19: Artificial frame with different contrast to back and different shift motion to down side (720x480 pixels).

For small shift (1 pixel) the proposed algorithm does not detect feathering, which is almost invisible to human eye. For shift level of 2 pixels, the detection is improved, and it is possible to verify that the contrast to back increases the proposed algorithm detection capacity.

Another artificial frame as showed on Figure 20, where the objects have the same size and present motion on diagonal direction, with different pixel shifts and different levels of contrast to background (500x400 pixels).

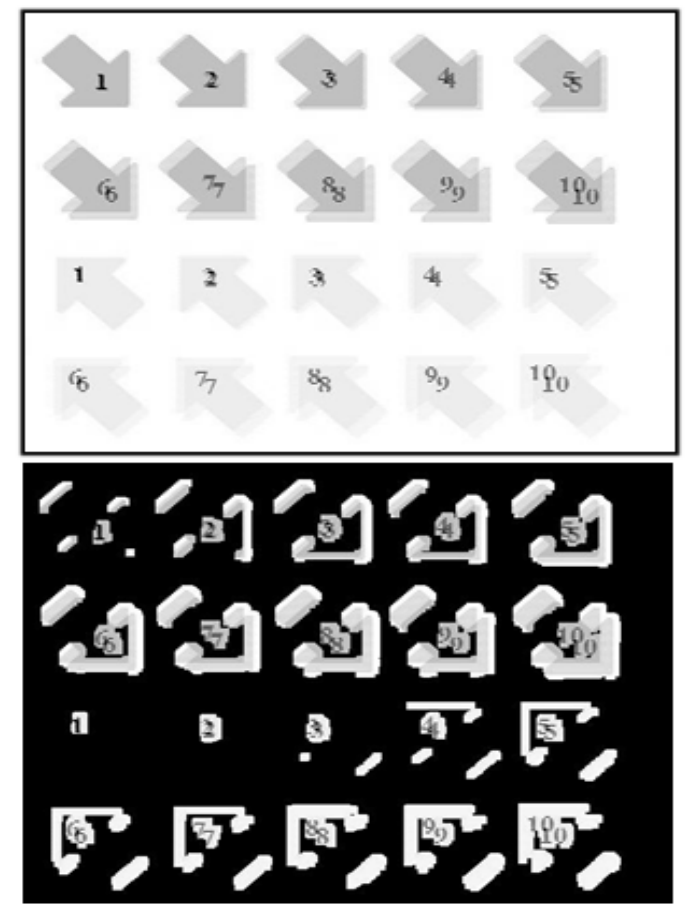

Figure 20: Artificial frame with objects in diagonal motion with different pixel shifts and different contrast to background. 
Comparing results on Figure 19 and Figure 20, the algorithm can be considered invariant to the parameters motion direction and contrast to background.

In the next section, the conclusions regarding the proposed method are presented.

\section{CONCLUSIONS}

The preliminary hypothesis about the periodic waveform like nature of feathering effect was verified.

Based on this preliminary hypothesis verification, the proposed feathering effect detection algorithm of interlaced video frames is effective and is capable of identifying defects in several situations and objects motion characteristics, contributing to the advancement in terms of performance of methods of video de-interlacing.

Conducted tests showed the proposed algorithm is invariant to objects motion direction and the contrast of object pixel level to background. It is also invariant to image resolution and object size.

As expected from the detector, the main sensitivity of the proposed algorithm is related to motion shift level in pixels, as the feathering effect is to visual discomfort.

Another advantage presented by the proposed algorithm is that by just adjusting one simple parameter, represented by the effective accumulated value threshold, it is possible to change the detection level considered as the point of visual discomfort for the human eye on specific scene situations.

In future work it is important to investigate the performance of the algorithm in videos with camera motion situations, and objects occlusion conditions.

It is intended to combine the proposed algorithm with deinterlacing strategies and evaluate the performance improvements, so as to obtain enough image quality to be worth to include it in video signal processors for TV sets.

\section{REFERENCES}

[1] Martins, A.L., Rodrigues, E.L.L., Paiva, S.M.V. 2015. International Journal of Computer Applications, vol. 111, no. 1 , pp 0975-8887.

[2] Bellers, E.B. and De Haan, G. 2000. De-interlacing: A key technology for scan rate conversion," Elsevier, Amsterdam.

[3] Doyle, T., Looymans, M. 1990. Progressive scan conversion using edge information. Signal Processing of HDTV, II, Elsevier, pp. 711-721.

[4] Jeong, J., Jeon, G. and Wang, J. 2014. De-Interlacing Algorithm Using Weighted Least Squares. IEEE Trans. On Cir. \& Syst. For Video Tech, vol. 24-, no. 1, pp. 3948.

[5] Chang, Y.L, Lin, S.F., Chen, C., Chen, L.G. 2005. Video De-Interlacing by Adaptive 4-Field Global/Local Motion Compensated Approach. IEEE Trans. On Circuits and Systems for Video Technology, vol. 15, no. 12, pp 15691582 .

[6] Jeon, G., Anisetti, M., Bellandi, V., Jeong, J. 2007. Fuzzy rule-Based Edge-Restoration Algorithm in HDTV Interlaced Sequences. IEEE Trans. On Consumer Electronics, vol. 534, no. 2, pp 725-731.

[7] Fan, Y.C, Chung, C.H. 2009. De-interlacing Algorithm Using Spatial-temporal Correlation-Assisted Motion Estimation. IEEE Trans. On Circuits and Systems for Video Tech., vol. 19, no. 7, pp. 932-944.

[8] Sreekanth, S., Chakrapani, Y., Sri, K.U. 2011. Motion Adaptive Compensation Approach for Deinterlacing of Video Sequences. International Journal of Computer Applications, vol. 15, no. 3, pp. 0975-8887.

[9] PC Magazine Online. About feathering effect. PC Magazine web site, Ziff Davis, LLC. http://www.pcmag.com/encyclopedia/term/43070/feather ing/. (2016).

[10] Chen, Y.R., Tai, S.C. 2009. True Motion-Compensated De-Interlacing Algorithm. IEEE Trans. On Circuits and Systems for Video Tech., vol. 19, no. 10, pp. 1489-1498.

[11] The MathWorks, Inc. About MatLab platform. Mathworks web site. http://www.mathworks.com/products/matlab/. (2016). 